\title{
Intranasal delivery of mesenchymal stem cell secretome repairs the brain of Alzheimer's mice
}

\author{
Giulia Santamaria ${ }^{1}$ Edoardo Brandi ${ }^{1}$ Pietro La Vitola ${ }^{1}$. Federica Grandi ${ }^{1} \cdot$ Giovanni Ferrara $^{2}$. \\ Francesca Pischiutta ${ }^{1}$ - Gloria Vegliante ${ }^{1}$ - Elisa R. Zanier ${ }^{1}$ - Francesca Re $^{3}$ - Antonio Uccelli ${ }^{2,4}$ - Gianluigi Forloni ${ }^{1}$. \\ Nicole Kerlero de Rosbo ${ }^{2} \cdot$ Claudia Balducci $^{1}$
}

Received: 11 July 2019 / Revised: 9 July 2020 / Accepted: 10 July 2020 / Published online: 23 July 2020

(c) The Author(s) 2020. This article is published with open access

\begin{abstract}
The multiplicity of systems affected in Alzheimer's disease (AD) brains calls for multi-target therapies. Although mesenchymal stem cells (MSC) are promising candidates, their clinical application is limited because of risks related to their direct implantation in the host. This could be overcome by exploiting their paracrine action. We herein demonstrate that in vivo systemic administration of secretome collected from MSC exposed in vitro to AD mouse brain homogenates (MSCCS), fully replicates the cell-mediated neuroreparative effects in APP/PS1 AD mice. We found a complete but transient memory recovery by 7 days, which vanished by 14 days, after a single MSC-CS intravenous administration in 12-month or 22-24-month-old mice. Treatment significantly reduced plaque load, microglia activation, and expression of cytokines in astrocytes in younger, but not aged, mice at 7 days. To optimize efficacy, we established a sustained treatment protocol in aged mice through intranasal route. Once-weekly intranasal administration of MSC-CS induced persistent memory recovery, with dramatic reduction of plaques surrounded by a lower density of $\beta$-amyloid oligomers. Gliosis and the phagocytic marker CD68 were decreased. We found a higher neuronal density in cortex and hippocampus, associated with a reduction in hippocampal shrinkage and a longer lifespan indicating healthier conditions of MSC-CS-treated compared to vehicletreated APP/PS1 mice. Our data prove that MSC-CS displays a great multi-level therapeutic potential, and lay the foundation for identifying the therapeutic secretome bioreactors leading to the development of an efficacious multi-reparative cocktail drug, towards abrogating the need for MSC implantation and risks related to their direct use.
\end{abstract}

Edited by N. Bazan

Supplementary information The online version of this article (https:// doi.org/10.1038/s41418-020-0592-2) contains supplementary material, which is available to authorized users.

$\triangle$ Claudia Balducci

claudia.balducci@marionegri.it

1 Department of Neuroscience, Istituto di Ricerche Farmacologiche Mario Negri IRCCS, via Mario Negri 2, 20156 Milan, Italy

2 DINOGMI, University of Genoa, Largo Daneo, 3, 16132 Genoa, Italy

3 School of Medicine and Surgery, Nanomedicine Center NANOMIB, University of Milano-Bicocca, Via Raoul Follereau 3, 20854 Vedano al Lambro, MB, Italy

4 Ospedale Policlinico San Martino IRCCS, Largo Rosanna Benzi, 10, 16132 Genoa, Italy

\section{Introduction}

Alzheimer's disease (AD) is the main form of dementia in the elderly [1], urgently calling for a cure. AD brains show deposited extracellular senile plaques enriched in $\beta$-amyloid (A $\beta)$ aggregates, and intracellular tau-enriched neurofibrillary tangles [2]. Neuropathology is complex and affects synaptic and cognitive function [3], as well as ion channels, mitochondrial function, and the vascular system [4]. AD brains are permanently inflamed, and released cytokines foster neurotoxicity culminating in gross brain atrophy $[5,6]$. Although $\mathrm{A} \beta$ remains the main recognized culprit of $\mathrm{AD}, \mathrm{A} \beta$-centric therapies likely fail because the complex pathology needs multi-target approaches.

Mesenchymal stem cells (MSC) hold great promise as an alternative, multi-level approach in the therapy of intractable neurodegenerative disorders. MSC exert direct neuroprotective/neuroreparative effects through the release of neurotrophic factors and potent immunomodulatory 
properties [7]. Notably, MSC are the only stem cells endowed with anti-amyloidogenic activities [8]. MSC therapeutic potential has been documented in various $\mathrm{AD}$ animal models with data showing anti-inflammatory, antiamyloid properties, and memory recovery [8, 9] and is being considered for several neurological diseases [10].

In spite of many advantages of MSC-based therapy, various challenges limit their clinical application, including: (i) invasive cell isolation process, (ii) loss of potency, (iii) limited lifespan, (iv) huge expansion costs [11], as well as the possibility, albeit low, of uncontrollable cell proliferation. It is well-accepted that infused MSC act through paracrine mechanisms by releasing bioactive components in their secretome when exposed to an injured environment, rather than through direct engraftment. In vitro and in vivo studies have shown both neuroprotective and antiinflammatory effects exerted by MSC secretome itself in various disease models $[11,12]$, but not yet in $\mathrm{AD}$ mice.

We herein demonstrate that the secretome derived from MSC pre-conditioned in vitro in an $\mathrm{AD}$ environment (MSC-CS) fully replicates multiple neuroreparative activities exerted by implanted MSC themselves. Furthermore, we establish a preclinical, non-invasive and sustained treatment protocol leading to a reversal of functional and structural damage in aged $\mathrm{AD}$ mice. These findings hold enormous translational potential, since they provide the opportunity to circumvent the direct implantation of MSC and the limitations related to their clinical application.

\section{Results}

\section{MSC-CS rapidly restores memory in 12-month-old APP/PS1 mice, albeit transiently}

It is hypothesized that the crosstalk between MSC and the "injured" environment may be vital for the release of protective factors by MSC. Thus, we tested whether exposure of MSC to AD brain homogenate would have stimulated MSC to release a neuroreparative secretome. We, thus, compared the therapeutic efficacy of the secretome derived from MSC grown in standard conditions (MSC-UCS) with that derived from MSC exposed to brain homogenate from APP/PS1 mice (MSC-CS). The MSC-CS dose selected was that corresponding to $1 \times 10^{6} \mathrm{MSC}$ inducing memory recovery when IV injected in 11-month-old APP/PS1 mice (Fig. S1).

MSC-CS or MSC-UCS were intravenously (IV) injected in 12-month-old APP/PS1 mice tested 7 days post-injection in the novel object recognition memory test (NORT) (experimental design in Fig. 1a). A single MSC-CS IV administration induced a significant memory recovery in APP/PS1 mice. Of note, MSC-UCS administration was not effective (Fig. 1b). The efficacy of MSC-CS was fully confirmed in a second experiment, also showing that MSCCS did not affect memory of WT mice (Fig. 1c).

When mice were retested in the NORT 14 days posttreatment, the overall effect of MSC-CS on memory was lost in $50 \%$ of MSC-CS-treated mice, thus indicating a

A
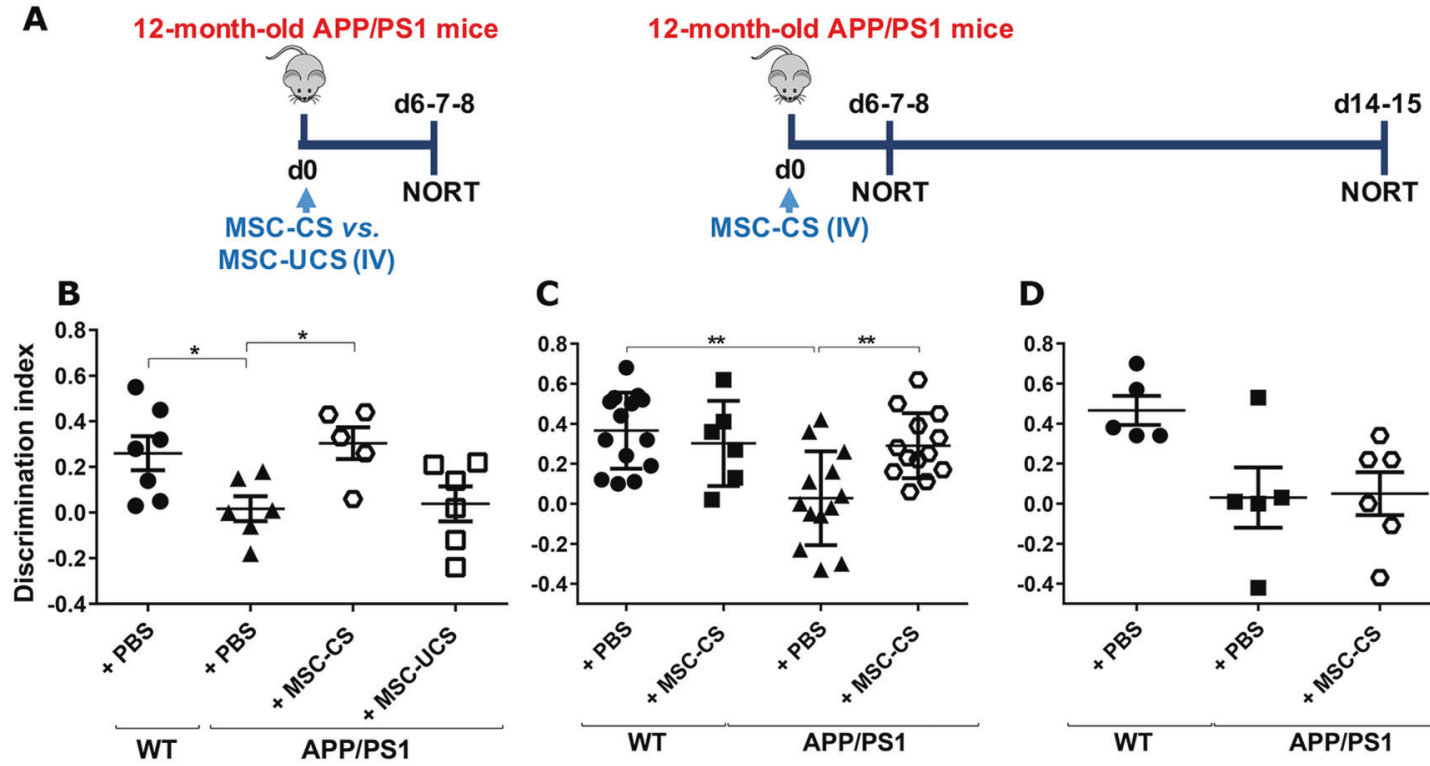

MSC-CS (IV)
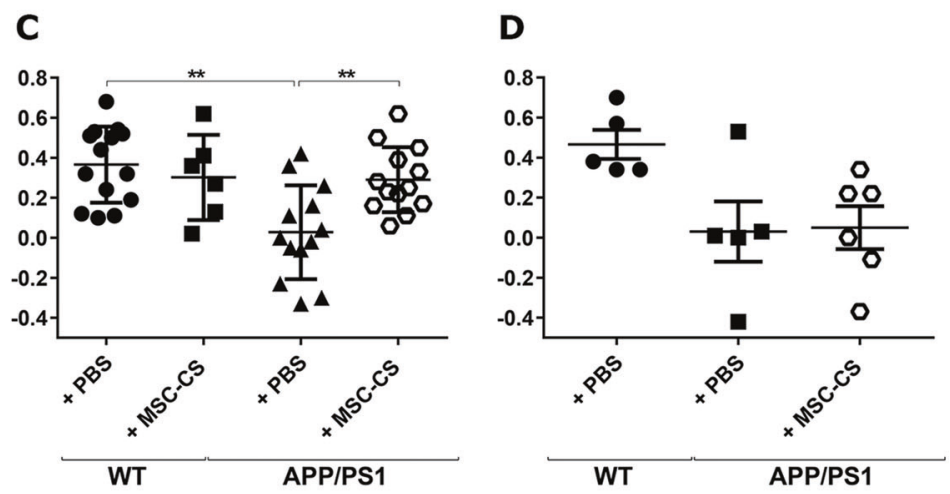

Fig. 1 One IV injection of MSC-CS, but not MSC-UCS, transiently restores memory in 12-month-old APP/PS1 mice. a Experimental design. b Comparison of the discrimination index (DI) of APP/PS1 mice and their age-matched WT littermates receiving either PBS, MSC-CS or MSC-UCS and tested in the NORT. DI of WT and
APP/PS1 mice treated with PBS or MSC-CS and tested in the NORT 7 days (c) and 14 days (d) post-injection. Data are expressed as scatter plots with mean \pm SEM. One-way ANOVA in $\mathbf{b}$ and $\mathbf{d}$. Two-way ANOVA in $\mathbf{c}, * P<0.05 ; * * P<0.01$ Tukey's multiple comparison post-hoc test. 
transient efficacy of single-dose MSC-CS administration (Fig. 1d).

\section{A single MSC-CS IV injection is sufficient to decrease amyloidosis and neuroinflammation}

Immunohistochemical analysis for amyloid plaques (Fig. 2b) on brain tissue from 12-month-old APP/PS1 mice treated with MSC-CS (experimental design in Fig. 2a) and plaque quantification (Fig. 2c) showed a 30\% significant reduction in the number of total hippocampal and cortical $\mathrm{A} \beta$ plaques.

$\mathrm{A} \beta$ plaques in patients and mice are intimately surrounded by activated glial cells with a double sword action promoting plaque clearing $[6,13]$, but also neurotoxicity and memory impairment [14]. We thus evaluated the extent of hippocampal inflammation, a crucial brain area involved in recognition memory [15] and with prominent gliosis in
APP/PS1 mice at this age. A co-localization of plaques and microglia well describing MSC-CS effect on the microglia pan marker IBA1 is shown in Fig. 2d. MSC-CS-treated APP/PS1 mice showed a significant reduction in IBA1 positivity (Fig. 2e), with residual cells displaying a resting state-like morphology. Treated APP/PS1 mice also showed a significant reduction in the CD68-marked area (Fig. 2f, g), a lysosomal glycoprotein associated with phagocytic function and lysosomal trafficking [16, 17], suggesting that spared plaques neither recall microglia nor exert a proinflammatory action.

We then assessed the effect of MSC-CS on astrogliosis, another neuroinflammatory hallmark in AD patient [14] and mouse [5] brains, by measuring the expression of the antiglial fibrillary acidic protein (GFAP) through histology. GFAP-marked area was not affected by the treatment (Fig. 3a, b). Notably, when the two most representative cytokines of $\mathrm{AD}$, interleukin- $1 \beta$ (IL-1 $\beta$ ) and tumor necrosis
Fig. 2 A single IV injection of MSC-CS reduces brain amyloidosis and microglial activation. a Experimental design. b Confocal images of the hippocampal area marked with the $6 \mathrm{E} 10$ anti-A $\beta$ antibody, evidencing deposited amyloid plaques. c Plaque quantification. d Confocal images of the hippocampal area marked with both $6 \mathrm{E} 10$ for plaques and IBA1 for microglial cells. e IBA1marked area quantification. $\mathbf{f}$ Hippocampal area immunostained for the phagocytic marker CD68 quantified in g. Data are expressed as scatter plots with mean \pm SEM. $* P<0.05$; $* * P<$ 0.01 Student's $t$ test.
A 12-month-old APP/PS1 mice
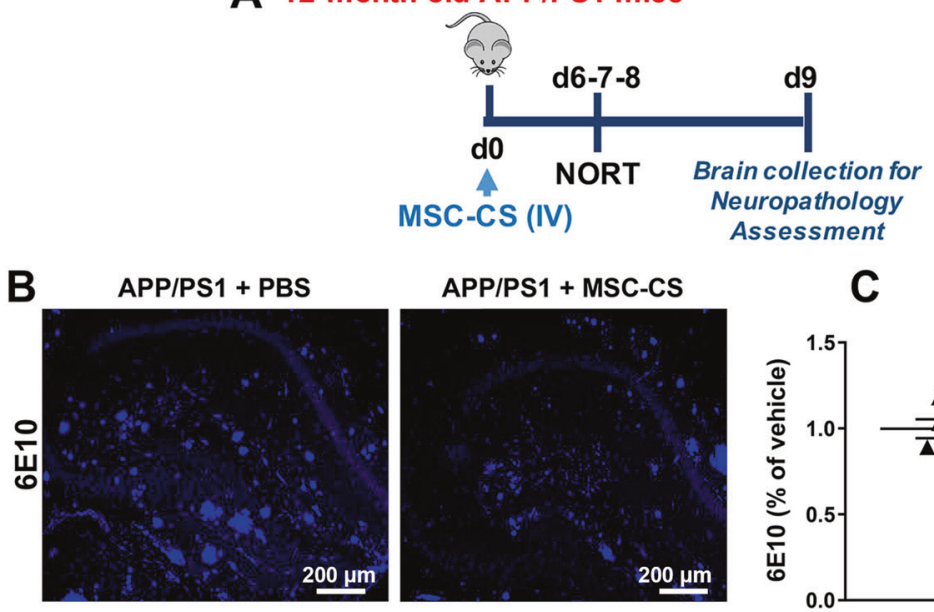

APP/PS1 + MSC-CS

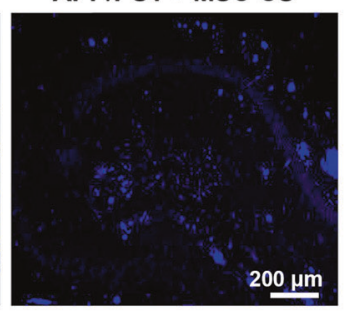

C

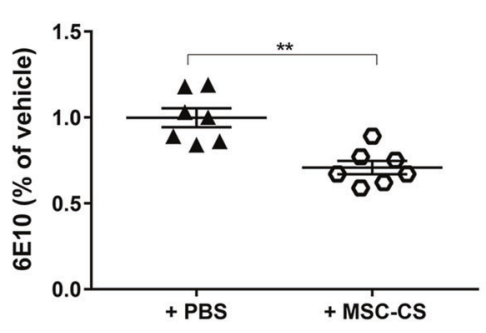

D
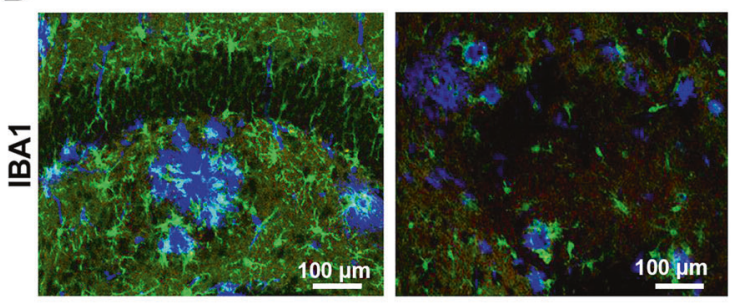

E

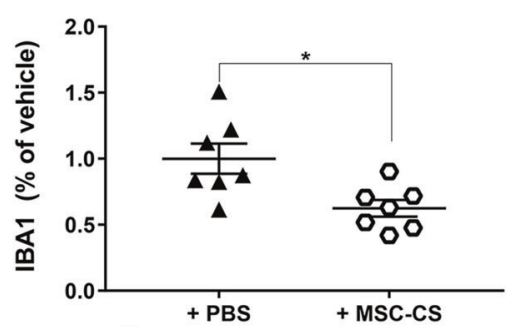

$\mathbf{F}$





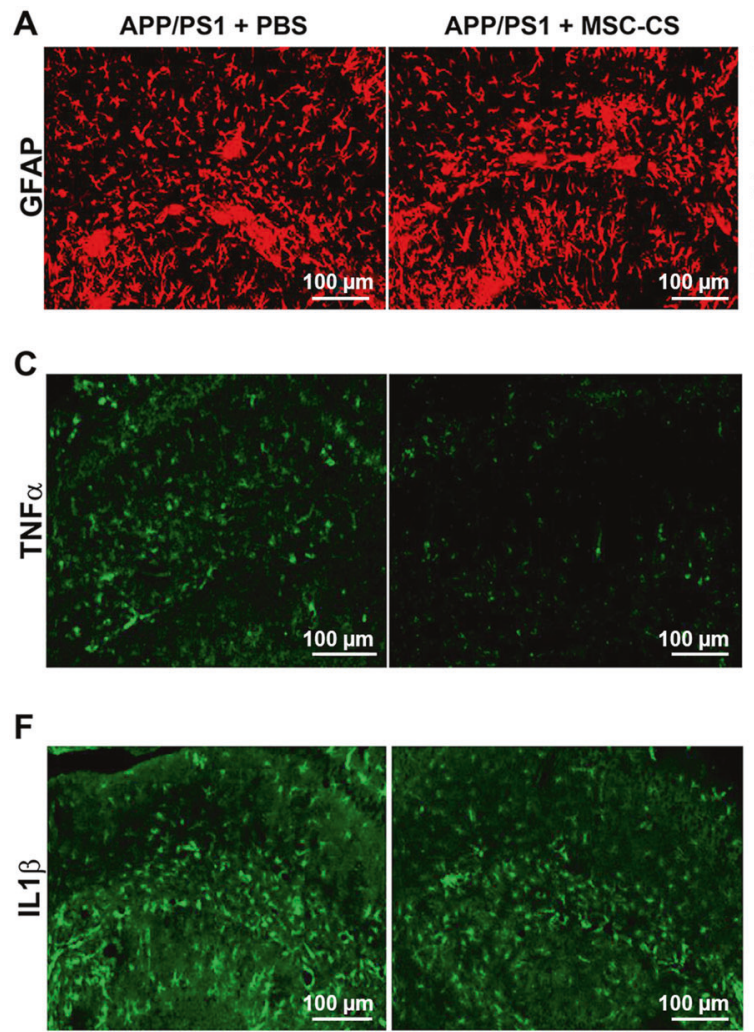

Fig. 3 Single IV injection of MSC-CS improves the inflammatory phenotype of astrocytes. a Confocal images of hippocampal slices from MSC-CS-treated and PBS-treated mice immunostained for GFAP. b GFAP-marked area quantification evidencing the degree of astrogliosis between treatment groups. c Hippocampal slices immunostained for $\mathrm{TNF} \alpha$ and relative quantification in d. e Merge of

factor- $\alpha(\mathrm{TNF} \alpha)$ [18], were analyzed, we found a statistically significant $30 \%$ decrease in hippocampal $\mathrm{TNF} \alpha$ expression (Fig. 3c, d) between MSC-CS- and PBS-treated APP/PS1, whereas the expression of IL-1 $\beta$ was unchanged (Fig. 3f, g). As both cytokines are mainly expressed by astrocytes in APP/PS1 mice (Fig. 3e and h), these data suggest that MSC-CS treatment partially reduced the proinflammatory astrocyte A1 phenotype [19].

\section{A single IV injection restores memory in 22-month- old APP/PS1 mice, but fails to improve neuropathology}

In order to assess MSC-CS efficacy at a more severe stage of $\mathrm{AD}$, we replicated our study in 22-month-old APP/PS1 mice. Again, a single IV injection of MSC-CS restored APP/PS1 recognition memory in the NORT 7 days posttreatment (Fig. S2A). Unexpectedly however, it did no affected amyloidosis or neuroinflammation (Fig. S2B, C), thus indicating that a single injection is sufficient to restore
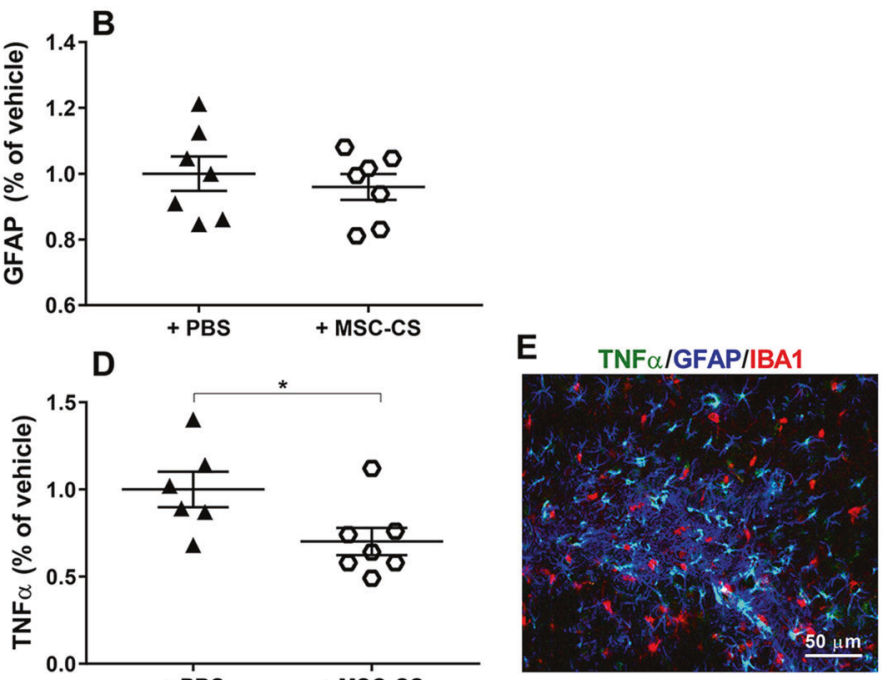

G

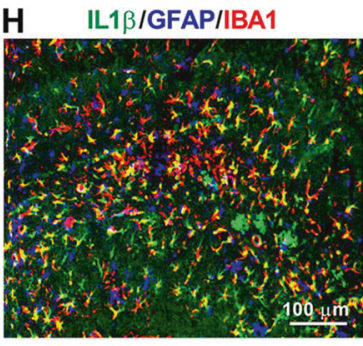

hippocampal immunostaining for TNF $\alpha$-GFAP-IBA1. f Hippocampal immunostaining for IL1 $\beta$ quantified in $\mathbf{g}$; $\mathbf{h}$ Merge of hippocampal immunostaining for IL1 $\beta$-GFAP-IBA1. Quantification data are expressed as scatter plots with mean \pm SEM. $* P<0.05$, Student's $t$ test.

memory at this advanced age, but fails to modify other crucial biomarkers.

\section{Continuous, weekly, intranasal treatment with MSC- CS succeeds in imparting sustained memory recovery and repairing the neuropathology in 25- month-old APP/PS1 mouse brain}

The transient functional recovery upon MSC-CS treatment and no improvement in neuropathology in aged mice suggested the need for a regular treatment to optimize the protocol. Due to limitations linked to repeated IV injections, we chose to exploit the intranasal (IN) route, which is noninvasive and offers the advantage of direct MSC-CS brain delivery via olfactory route [20].

Twenty-two-month-old APP/PS1 mice received a first IN administration of MSC-CS and were tested 7 days later in the NORT (Fig. 4a), to verify if a single IN administration could replicate the memory recovery obtained with one IV injection. The treatment only partially rescued mouse 
A 22-month-old APP/PS1 mice

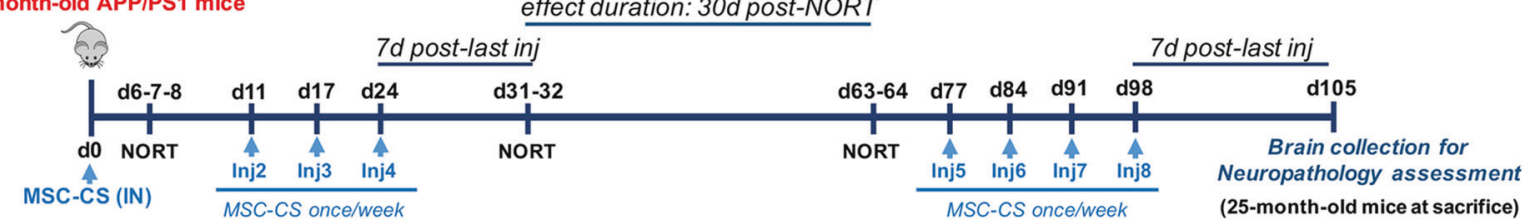

B

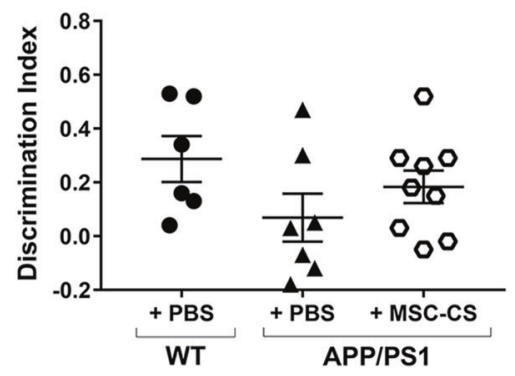

$\mathbf{E}$

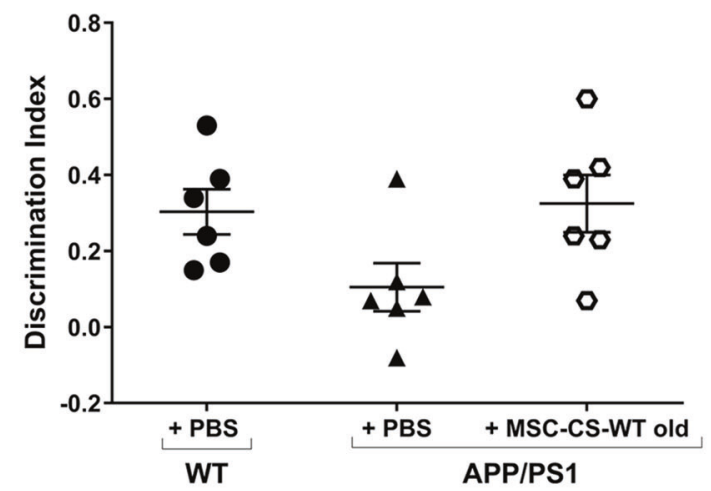

Fig. 4 Repeated IN delivery of MSC-CS significantly restores memory whereas MSC-CS-WT old or young has partial or no effect, respectively. a Experimental design for MSC-CS treatment. DI of the recognition memory task of mice tested 7 days after 1 (b) or 4 (c) IN administrations, or 39 days after suspension of MSC-CS treatment (d). e DI of the recognition memory task of mice tested

memory (Fig. 4b), and was thus continued once weekly for one month. NORT 7 days after the end of treatment showed that memory was fully restored in APP/PS1 mice (Fig. 4c).

To verify if a regimen of regular administration is needed to guarantee the long-lasting functional effect, we re-tested these mice 39 days after treatment suspension. MSC-CStreated mice showing a complete memory recovery oneweek post-treatment were newly impaired (Fig. 4d) indicating that a sustained MSC-CS administration protocol is needed to preserve functional effects.

While we demonstrated that conditioning of MSC with $\mathrm{AD}$ brain homogenate is necessary to license the therapeutic features of the secretome, we cannot exclude that this effect might be related to inflammatory molecules present in the normal aged brain. Indeed, it is well-known that normal aging is associated with neuroinflammation [21, 22]. Accordingly, we have compared the licensing effect on
D
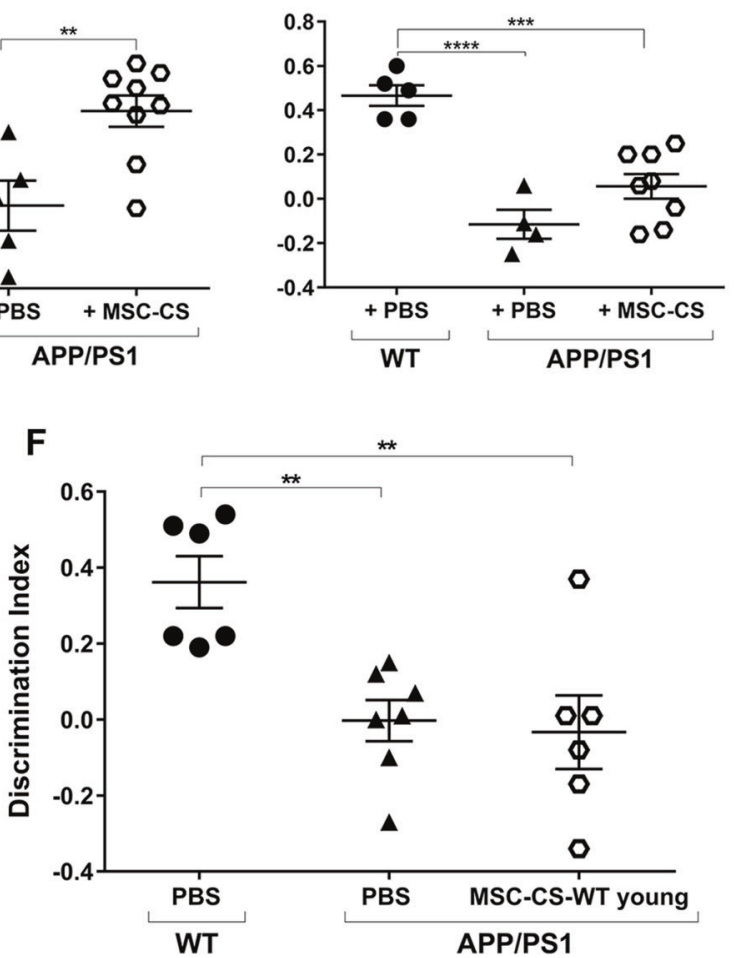

7 days after 4 IN administrations of MSC-CS-WT old. $\mathbf{f}$ DI of the recognition memory task of mice tested 7 days after 4 IN administrations of MSC-CS-WT young. Data are expressed as scatter plots with mean \pm SEM. One-way ANOVA; $* * P<0.01 ; * * * P<0.001$; $* * * * P<0.0001$, Tukey's multiple comparison post-hoc test.

MSC of brain homogenate from old WT mice (20 months of age) with that of young WT mice (6 months of age). Old APP/PS1 mice (22-25 months of age) were treated as above (4 weekly IN administrations) with the resulting MSC-CS (MSC-CS-WT old or MSC-CS-WT young). Upon testing in the NORT, there was an almost significant memory improvement in old APP/PS1 mice treated with MSC-CSWT old $(P=0.063$; Fig. 4e). In contrast, MSC-CS-WT young had no beneficial effect on memory loss in old APP/ PS1 mice (Fig. 4f).

\section{Repeated IN MSC-CS treatment reduces neuropathology in 25-month-old APP/PS1 mice}

As shown above, withdrawal of treatment led to reestablishment of memory impairment assessed at 39 days after treatment suspension in old APP/PS1 mice (Fig. 4d). 

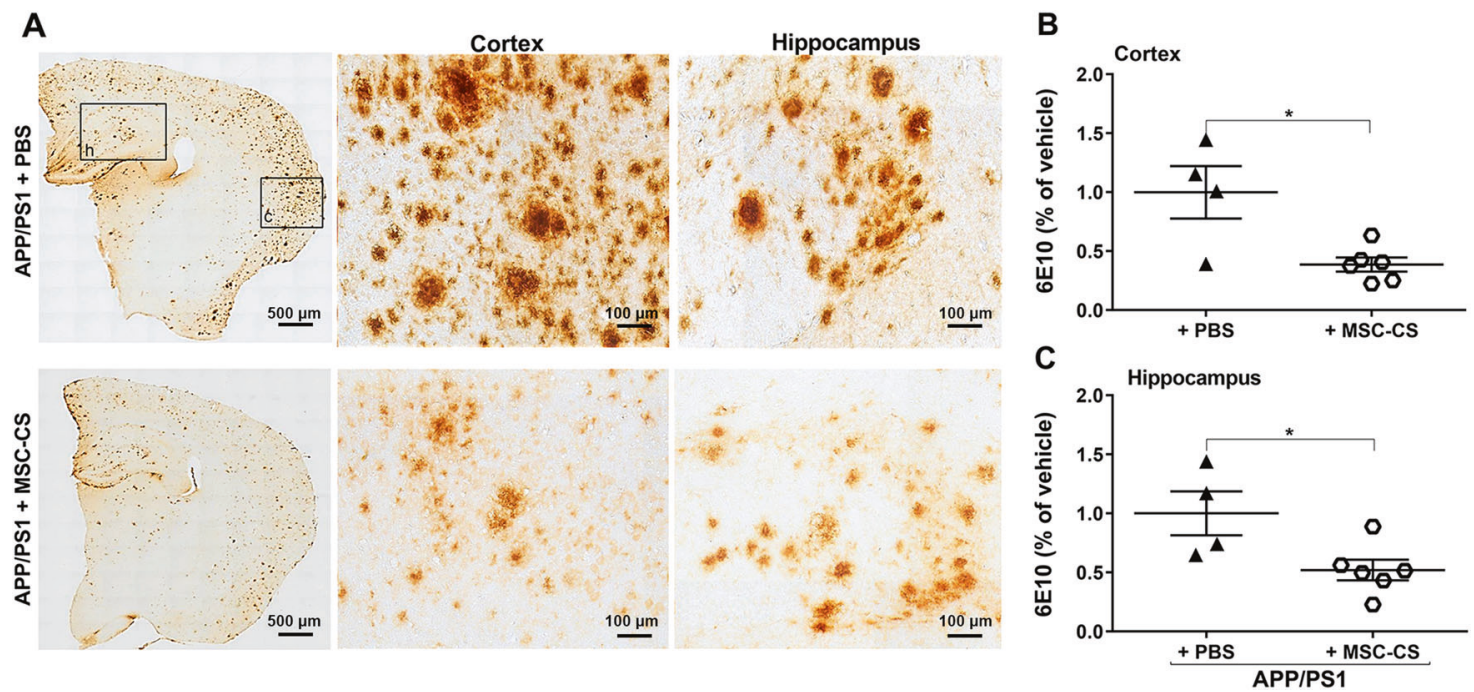

Fig. 5 Repeated IN delivery of MSC-CS significantly decreases brain amyloidosis. a $6 \mathrm{E} 10$ immunostaining comparing brain plaque load between MSC-CS- and PBS-treated mice (left panels). In the middle and right panels, higher magnification of plaques in cortex (c) and hippocampus (h) showing MSC-CS-mediated changes in terms of aggregate density, and absence of the central plaque core. b, $\mathbf{c}$ Plaque quantification in the cortex and the hippocampus. Quantitative data are expressed as scatter plots with mean \pm SEM. $* P<0.05$, Student's $t$ test.

the A11 anti-A $\beta O$ antibody to assess if MSC-CS had modified their load. We found that APP/PS1 mice treated with MSC-CS appeared to have a much lower $A \beta O$ load around plaques (Fig. 7a, b), which might explain the decrease in the phagocytic commitment of microglia and in the extent of neuroinflammation. Despite multiple attempts, we were not able to actually quantify the $\mathrm{A} \beta \mathrm{O}$ specifically; indeed, although the oligomers show a darker, distinguishable staining, they overlap with the plaque halo, which could not be subtracted with Fiji software.

Double-staining A11-6E10 ascertained that the A11 antibody was not reacting with the entire plaque in an unspecific manner (Fig. S3A), but it marked only small aggregates surrounding plaques and some positivity was detectable also far from plaques (Fig. S3A). Notably, superresolution microscopy revealed that $\mathrm{A} \beta \mathrm{O}$ s are mainly engulfed in the lysosomes independently of the treatment (Fig. S3B).

\section{MSC-CS-treated 25-month-old APP/PS1 mice show increase neuronal density in both cortex and hippocampus and diminished hippocampal shrinkage}

Because of the well-known neuroprotective action of MSC, we investigated if the treatment affected neuronal density in the cortex and hippocampus of our mice, by comparing NISSL-stained brain slices from PBS-treated WT vs. PBSor MSC-CS-treated (8 IN) 25-month-old APP/PS1 mice. Through a well-established home-made macro [25], specifically counting neurons, we found that MSC-CS induced a 
A
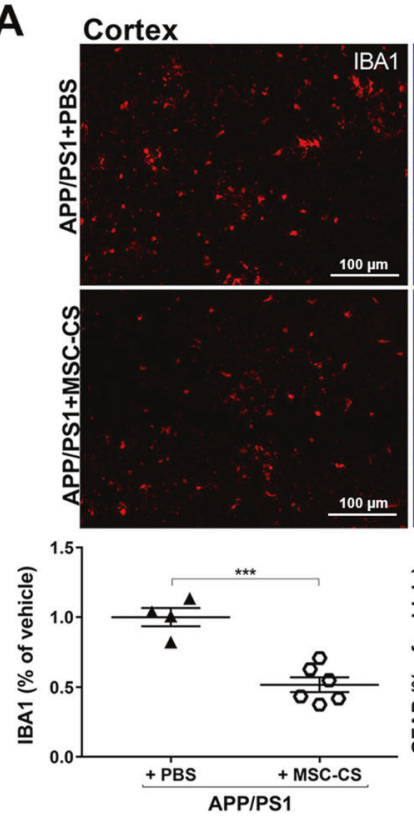

C

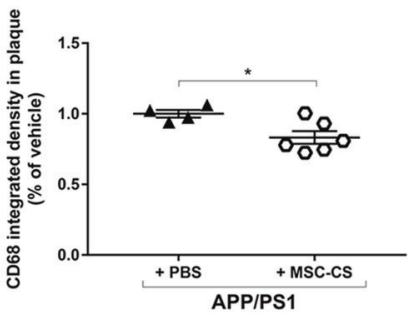

B
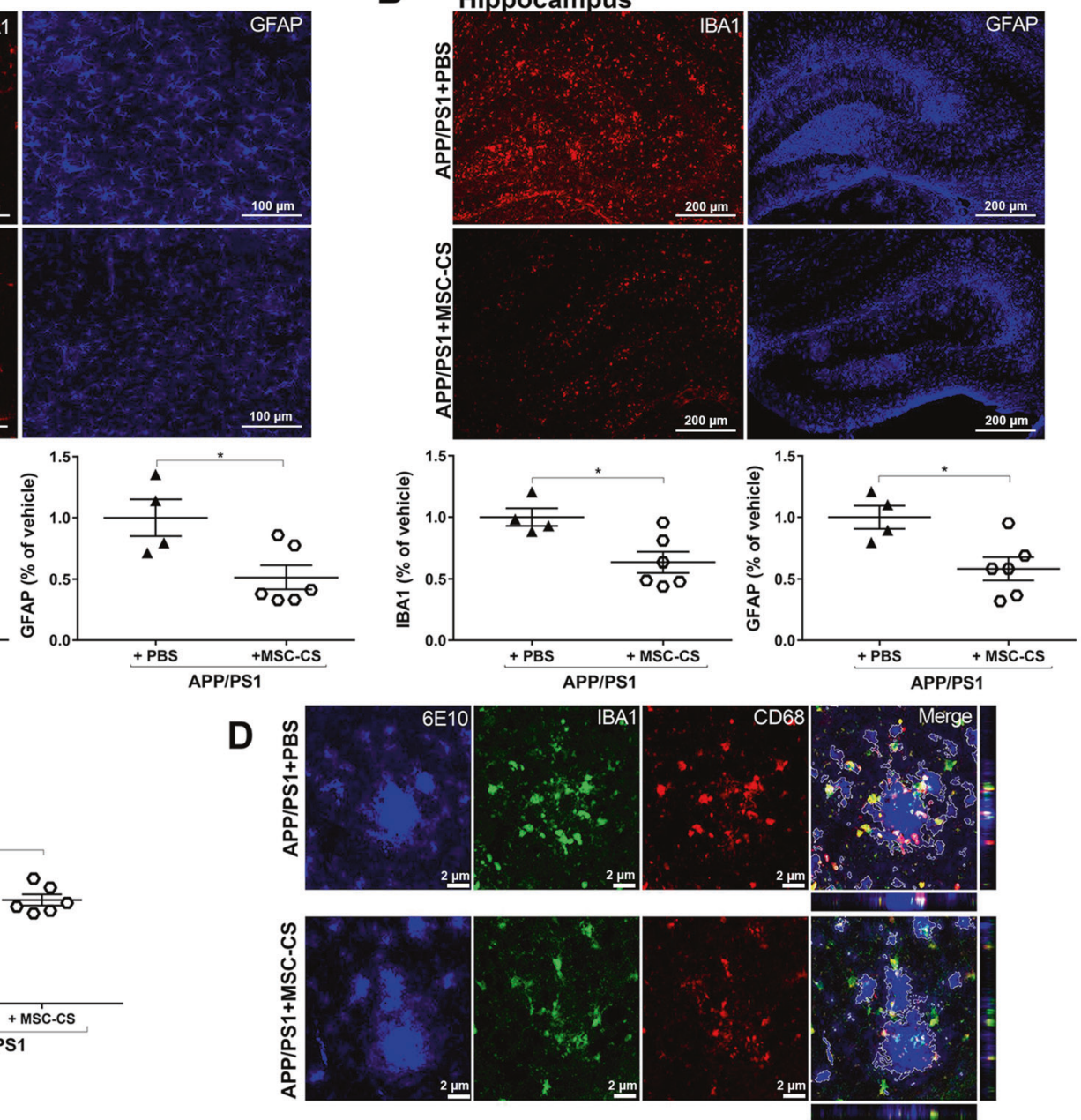

Fig. 6 Repeated IN injections of MSC-CS significantly reduce neuroinflammation. a, b IBA1 and GFAP immunostaining showing the extent of gliosis in the cortex and the hippocampus between PBSand MSC-CS-treated (8 IN) 25-month-old APP/PS1 mice (upper panels). Quantification of the IBA1- and GFAP-marked areas in the cortex and the hippocampus, respectively (lower panels). c CD68

significant $10 \%$ increase in neuronal number in the whole cortex (Fig. 8a). We observed an even higher, significant $25 \%$ increase in the perirhinal (PRH) and entorhinal (EC) cortex (Fig. S4), more critically affected in AD pathology [26] and involved in recognition memory [27], respectively.

In the hippocampus we measured the CA1 and dentate gyrus (DG) layer thickness. In PBS-treated APP/PS1 mice both layers were significantly thinner $(-25.6 \% \mathrm{CA} 1 ;-15 \%$ DG) than in WT mice, whereas in MSC-CS-treated APP/ PS1 mice, we appreciated a significant increment at both levels $(+17 \%$ CA $1 ;+21 \%$ DG) as compared to PBS-treated mice (Fig. 8b, c).

In addition, as read-out of hippocampal atrophy, we also systematically measured the neuropil thickness between the CA1 and DG subfields at 3 different levels (M1, M2, M3) in the same slice, and across the dorso-ventral hippocampal quantification in plaques. d Immunofluorescence images of plaques (6E10), CD68 and IBA1 expression, and CD68-IBA1 co-localization (Merge) around plaques between treatment groups. Quantitative data are expressed as scatter plots with mean \pm SEM. $* * * P<0.001 ; * P<$ 0.05 , Student's $t$ test.

extension (brain anterior-posteriority from -1.90 to -2.70 from bregma). Hippocampal atrophy was particularly evident in PBS-treated APP/PS1 mice compared to WT (Fig. 8d), as confirmed by a significant reduction in M1 $(-24.6 \%)$, M2 $(-27.8 \%)$ and M3 $(-26.3 \%)$ which was recovered by the treatment (Fig. 8e). We found that MSCCS-treated mice had a significantly higher M2 neuropil thickness $(+11.4 \%)$ and an almost significantly higher M3 $(+11.4 \%)$ compared to that of WT mice. We did not detect any changes at the M1 level (Fig. 8d, e).

The neuroprotective effect of MSC-CS was corroborated by some upregulation, albeit not significant, of the mRNA expression of growth factors, such as brain-derived neurotrophic factor (BDNF), nerve growth factor (NGF) and growth-associated protein 43 (GAP43), in the brain from MSC-CS-treated APP/PS1 mice (Table 1). In addition, we 


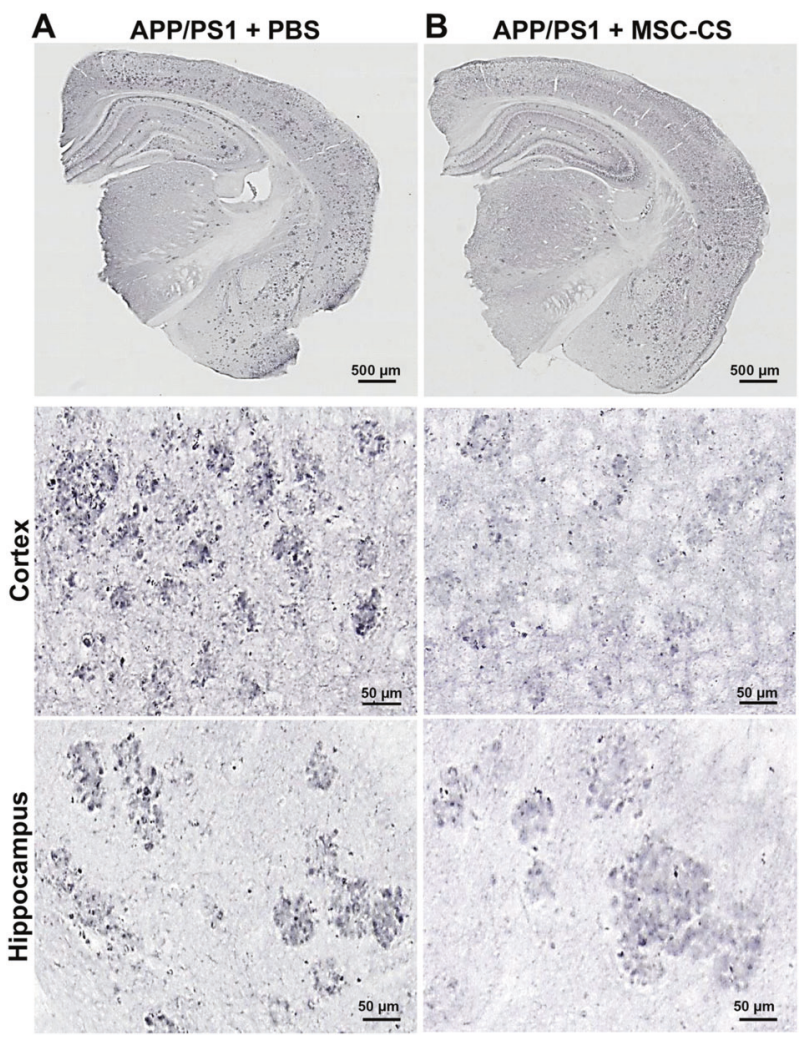

Fig. 7 Repeated delivery of MSC-CS appears to reduce AßO load around plaques in APP/PS1 mice. Images of APP/PS1 mouse brain slices immunostained with the anti-A $\beta O$ s A11 antibody showing the presence of A $\beta O$ s in PBS- (a) and MSC-CS-treated (8 IN) 25-monthold mice (b); upper panels. Middle and lower panels depict a higher magnification of plaques in the cortex and the hippocampus of APP/ PS1 mice showing $\mathrm{A} \beta \mathrm{O}$ distribution (darker dots).

found that mRNA expression of caspase 3, which reflects cellular apoptosis, was significantly reduced in MSC-CStreated APP/PS1 mice as compared to PBS-treated mice (Student's $t$ test $t_{8}=2,3 ; P=0.049$; Table 1). These results suggest that MSC-CS is promoting mechanisms of neuronal protection, together with a reduced apoptosis in APP/PS1 mice, which could explain, at least in part, the increase in the number of neurons we observed.

\section{APP/PS1 mouse lifespan is significantly increased by MSC-CS treatment}

Repeated administrations (8 IN) of MSC-CS clearly prolonged the lifespan of 25-month-old APP/PS1 mice with a significant $45 \%$ increase compared to PBS-treated APP/PS1 mice (Fig. 8f). Two out of 7 PBS-treated APP/PS1 mice died 31 days after the first injection and another one after 64 days. The remaining 4 were sacrificed after 74 days after the first injection because their health conditions were no longer optimal, and their blood, CSF and brains were collected. In contrast, of the 9 mice receiving MSC-CS, 1 died
93 days after the first injection, 2 after 100 days and the last 6 , still healthy, were sacrificed for neuropathology assessment 7 days after the end of the treatment, that is 105 days after the first injection.

\section{Discussion}

We herein demonstrated that systemic injections of the MSC-CS collected from mouse bone marrow-derived MSC fully replicate cell effects described in $\mathrm{AD}$ mice $[8,9]$. Sustained, MSC-CS intranasal administration in APP/PS1 mice fully restored mouse memory and impressively changed neuropathology at multiple crucial levels in very advanced disease stages. Although normal aging environment could foster the MSC-CS to assume an almost significant therapeutic effect, pre-conditioning of MSC to the specific AD microenvironment was fundamental to license them toward a significant neuroreparative, immunomodulatory phenotype. It was recently published that preconditioned MSC-derived exosomes restored memory and reduced inflammation when IV injected in APP/PS1 mice [28]. However, MSC were preconditioned through oxygen starving [28] rather than through a typical AD microenvironment as in our case. Our pre-conditioning more faithfully mimics the injured environment the cells would encounter, pointing to a specific $\mathrm{AD}$-associated protective MSC activation.

Memory recovery is one of the major clinical outcomes to achieve with AD therapy. We strikingly demonstrated that, as observed with MSC themselves [8, 9], one IV injection of MSC-CS rescued memory after only 7 days from the injection, in 12- and 22-month-old APP/PS1 mice. Memory recovery was only transient, likely suggesting that MSC-CS bioactive components repairing the mechanisms implicated in synaptic function and memory processing rapidly vanish after a single treatment, hence the need for sustained treatment.

In 12-month-old mice, a single injection of MSC-CS induced a $30 \%$ plaque reduction and glial activation. In the brain of $\mathrm{AD}$ patients and $\mathrm{AD}$ mice, plaques are surrounded by activated glia [29] releasing pro-inflammatory cytokines responsible for synaptic/cognitive dysfunction [30, 31], and neurotoxicity [5]. In MSC-CS-treated mice, residual plaques were smaller in size and bordered by only a few microglial cells. Further assessment of the lysosomal glycoprotein CD68, a marker of active microglial phagocytosis [16, 32], confirmed a decrease in the phagocytic commitment of microglia in treated mice, indicating that remaining plaques were no longer chemoattractant. We cannot yet establish which plaque clearance mechanism/s were stimulated by MSC-CS. Previous studies demonstrated that MSC co-cultured with $\mathrm{A} \beta$-treated neurons 

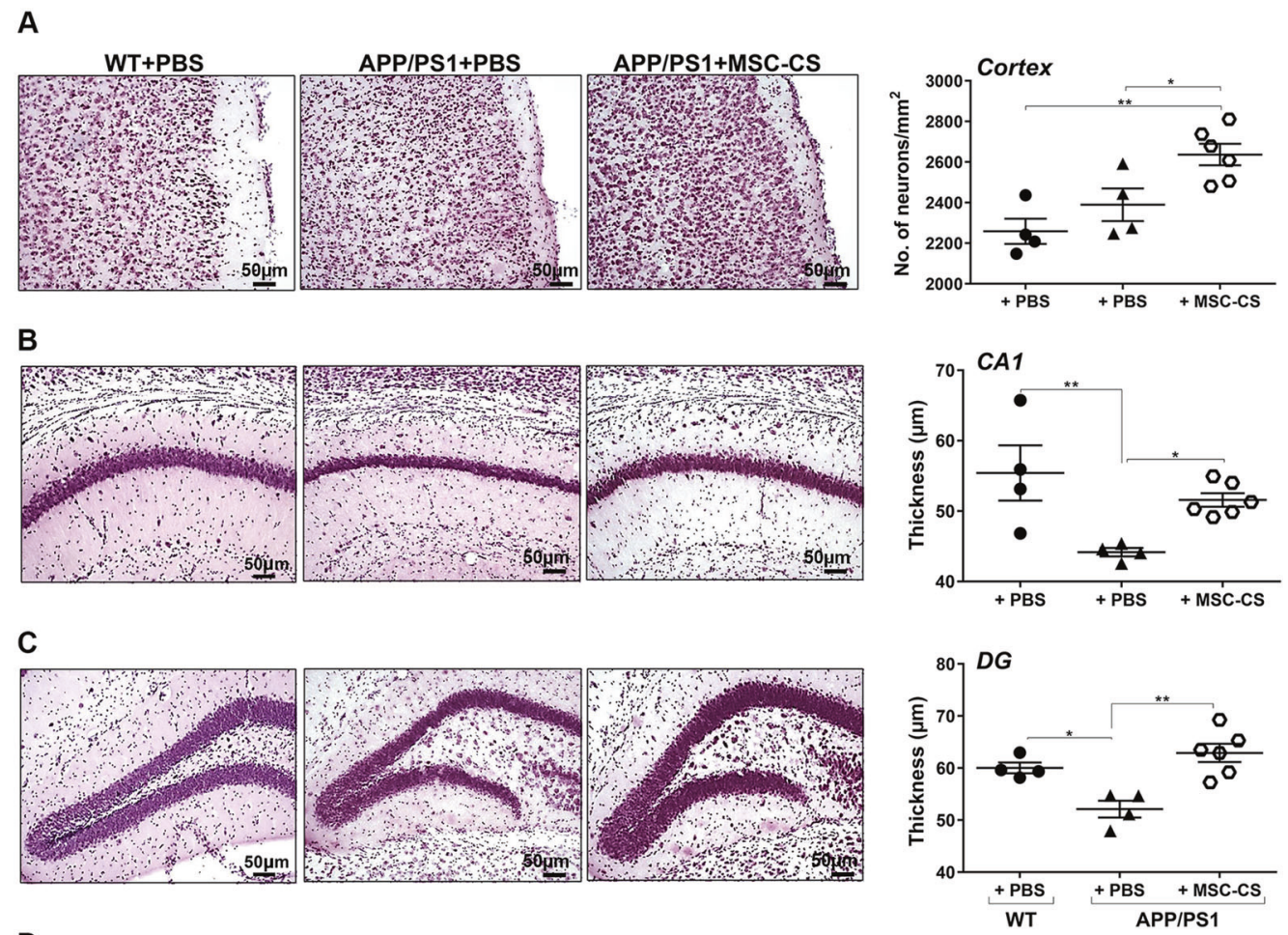

\section{D}
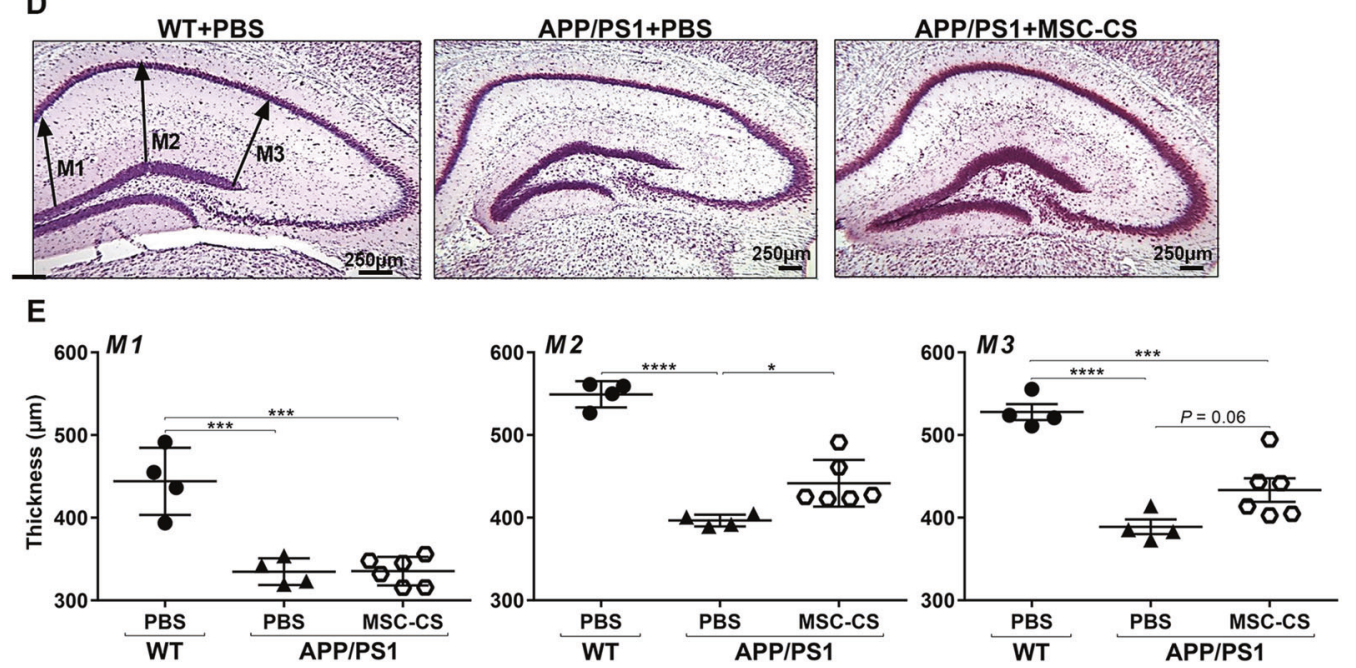

$\mathbf{F}$

‥ APP/PS1+PBS

+ APPIPS1+MSC-CS

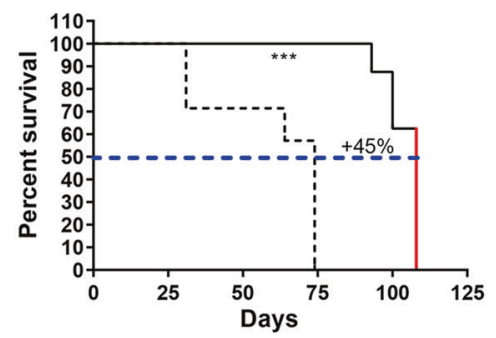

increased the number of LC3-II-positive autophagosomes co-localizing with lysosomal markers, an aspect which could be replicated by MSC-CS explaining plaque decrease
[33]. However, $A \beta$ clearance relies on the activation of several systems [34], thus dedicated studies are needed to clarify this issue. 
Fig. 8 APP/PS1 mice treated repeatedly with MSC-CS display a higher number of neuronal cells in the cortex and the hippocampus, reduced hippocampal atrophy, and increased survival. Left panels are representative NISSL-stained sections of the cortex (a) and cell layer thickness in the CA1 region of the hippocampus (b) and the dentate gyrus (DG) (c). Right panels show neuronal quantification thereof. d Images of NISSL-stained brain slices in which headed arrows at M1, M2 and M3 indicate where thickness was measured between the CA1 and DG hippocampal sub-regions. e Quantification of neuropil thickness for the 3 zones selected. Data are expressed as scatter plots with mean \pm SEM. One-way ANOVA; $* P<0.05$, $* * P<$ 0.01 ; $* * * P<0.001, * * * * P<0.0001$; Tukey's multiple comparison post-hoc test. f The graph describes mouse longevity comparison between MSC-CS-treated and PBS-treated APP/PS1 mice. The red line in APP/PS1 + MSC-CS indicates that these mice were sacrificed for experimental needs, but were still perfectly healthy. $* * * P<0.001$, Student's $t$ test.

We also demonstrated that MSC-CS partially reduced the pro-inflammatory astrocytic phenotype, by significantly decreasing their TNF $\alpha$ expression. New data demonstrated that the two glial cells are detrimentally interconnected with activated microglia promoting synapse loss, cognitive deficits [35], and an A1 astrocytic neurotoxic phenotype, with $\mathrm{TNF} \alpha$ as a crucial player [19]. Our findings demonstrate an association of MSC-CS-mediated positive effects leading to a reduction in plaque load and microglia activation, which resulted in restored memory and reduced A1 phenotype. However, this might be only partially true since one IV MSC-CS injection in aged mice did not modify brain neuropathology, despite an impressive memory recovery. Although we have no explanation for this, we assume that when the pathology is more severe, one MSC-CS injection is insufficient to modify neuropathology. In contrast, it appears that independently of plaque or glial changes, some secretome component/s directly act at neuronal level allowing regular memory processing. As aforementioned, this hypothesis is supported also by the fact that in the younger treated mice, memory recovery observed 7 days post-injection vanished 5 days later despite plaque reduction. These data are in agreement with the notion that plaque load is no longer considered as a correlate of synaptic and cognitive dysfunction, which has been functionally attributed to the soluble oligomeric $A \beta$ species [36].

Failure of a single IV MSC-CS injection to exert a beneficial effect at neuropathological level in older mice was overcome with a sustained IN treatment. IN administration is an alternative route [37], currently widely used for MSC or exosome administration with favorable outcomes [38-40].

We demonstrated in the NORT that MSC-CS induced full memory recovery after four IN injections; this functional effect was also subsequently confirmed under different treatment regimens in the Y-maze alternation test (data not shown). In addition, regular weekly MSC-CS
Table 1 Comparison among APP/PS1 experimental groups for BDNF, NGF, GAP43 and Caspase 3 mRNA expression.

\begin{tabular}{lcc}
\hline Gene & APP/PS1 + PBS & APP/PS1 + MSC-CS \\
\hline Bdnf & $-0.74 \pm 0.09$ & $-0.45 \pm 0.27$ \\
Ngf & $-0.64 \pm 0.19$ & $-0.43 \pm 0.06$ \\
Gap43 & $-0.28 \pm 0.42$ & $-0.19 \pm 0.06$ \\
Caspase 3 & $0.34 \pm 0.03$ & $0.20 \pm 0.05^{*}$ \\
\hline
\end{tabular}

$* P<0.05$, Student's $t$ test

administrations significantly reverted neuropathology in 25month-old APP/PS1 mice.

As an added value, we also proved that MSC conditioning strongly influences the therapeutic efficacy of the secretome. Indeed, lack of cell pre-conditioning failed to promote the therapeutic activity of the secretome. Moreover, the pre-conditioning effect could be age-related, reaching its maximal efficacy in presence of $\mathrm{AD}$ pathology. Indeed, MSC exposed to young WT mouse brain homogenate yielded MSC-CS with no reparative effect in 25month-old APP/PS1 mice, whereas MSC-CS-WT old exerted an almost significant reparative effect on the impaired memory of 22-month-old APP/PS1 mice. It is well known that normal aging is associated with neuroinflammation [21, 22]; our findings therefore suggest that inflammatory molecules, which are absent, or present in minimal amounts, in young brain are indeed relevant for the licensing of the neuroprotective effect of MSC that reaches its maximum expression when conditioning is induced with AD-related stimuli. A large number of studies clearly indicate that the environment in which MSC operate licenses their plasticity [41], and implicates diverse molecules. For example, an inflammatory environment mimicked by exposure to inflammatory cytokines is essential to promote the immunosuppressive function of MSC on T cells [42]. Although we cannot herein postulate what AD-related factor(s) are involved in priming our MSC, we assume that multiple pathology-related components such as the presence of $\mathrm{A} \beta$ aggregates, pro-inflammatory cytokines and chemokines could be implicated.

A healthier state in the 25-month-old APP/PS1 mice receiving sustained MSC-CS treatment with respect to agematched APP/PS1 mice receiving PBS was observed. The latter started to die about two months before the MSC-CS group and none of them survived until the end of the experiment. In contrast, 70\% of MSC-CS mice were still alive showing a wellbeing state and were sacrificed for neuropathological assessment. Increased survival was also described in a murine sepsis model treated with MSC preconditioned with staphylococcal enterotoxin B [43]. A clinical and neuropathological amelioration was observed in mice modeling multiple sclerosis treated either with MSC themselves [44, 45], or with their secretome [46]. In 
amyotrophic lateral sclerosis-affected mice, administration of MSC intravenously improved survival and motor function $[44,46,47]$.

IN-repeated MSC-CS administration in APP/PS1 mice from the age of 22 up to 25 months also induced a dramatic reduction in cortical and hippocampal plaque load, in the number of surrounding activated glial cells and in the expression of the phagocytic marker CD68. A further explanation for this latter outcome was the lower concentration of $\mathrm{A} \beta \mathrm{Os}$ in residual plaques. Plaques act as reservoirs of $A \beta O$ s, the smaller, soluble $A \beta$ species which freely circulate perturbing neuronal and glial activity $[4,23,48]$. Notably, in an A $\beta O$-induced acute mouse model [49], we showed that $A \beta O$ s specifically induce a reversible memory impairment through glial activation, and antiinflammatory drugs prevent it $[13,50]$. In our APP/PS1 mice, $A \beta O s$ were all mainly distributed within the plaque corona. Remarkably, super-resolution analysis found them all engulfed in CD68-positive lysosomes in both PBS- and MSC-CS-treated mice. Thus, we assume that microglial cells permanently engulf $\mathrm{A} \beta \mathrm{O}$ in the attempt to limit plaque growth or remove them from the brain; upon MSC-CS treatment, the lower $\mathrm{A} \beta \mathrm{O}$ concentration results in decreased microglia phagocytic commitment and reduced neuroinflammation. The ability of MSC-CS to lower the level of $\mathrm{A} \beta \mathrm{O}$ s is an incommensurable therapeutic potential in a translational prospective since $\mathrm{A} \beta \mathrm{O}$ s are the foremost target to counteract [23, 48].

Of note, MSC-CS also reduced hippocampal atrophy and neuronal damage in the brain of APP/PS1 mice. Brain atrophy is another core feature of $\mathrm{AD}$ [51], but to date no data were shown in this regard on the effects of MSC in mice. Patient brains are typified by gross neuronal loss. The entorhinal cortex is the first affected area followed by the subiculum and the CA1 sub-region of the hippocampus up to cortical areas [52]. We detected a significant hippocampal shrinkage and thinner pyramidal layers in both the CA1 and DG in PBS-treated APP/PS1 mice compared to WT, no longer detectable in APP/PS1 receiving MSC-CS. Accordingly, the neuropil thickness between the CA1 and DG pyramidal cell layer was significantly larger than in APP/ PS1 mice receiving PBS. Both CA1 and DG neuronal cell layers showed a significantly higher cell density compared to PBS-treated mice. A much higher number of cells in the cortex of MSC-CS-treated compared to WT and PBStreated APP/PS1 mice was also highlighted, accompanied by a decrease in mRNA expression of the apoptosis marker Caspase 3, and a trend in increased mRNA expression of nerve growth factors. These outcomes might depend on the ability of MSC-CS to protect neurons preventing their loss. Previous in vitro studies demonstrated that MSC-derived secretome promotes neuronal survival and increases metabolic activity of a stably transfected human cell model of
$\mathrm{AD}$ [53]. An increase in neurogenesis mediated by MSC was also described in A $\beta$-induced cells and AD mice [54]. It is also conceivable to assume that glial cell activation reduction might have prevented neuronal death. New investigations are ongoing to clarify these aspects in our mice.

In conclusion, we proved for the first time that the secretome derived from AD-conditioned MSC can fully replicate the neuroreparative actions previously described with MSC direct applications. The MSC-CS effects were impressive under longer treatments. Notably, besides plaque reduction the treatment also reduced their $\mathrm{A} \beta \mathrm{O}$ concentration, and despite the absence of cell implantation it abolished brain atrophy in crucial affected areas.

The paracrine action of MSC is becoming a certainty. The possibility to replace the use of stem cells with the safer application of those bioactive components cells would release in the host, represents an invaluable opportunity for the therapy of neurodegenerative diseases. Our study clearly proves the great therapeutic potential of MSC-CS and poses the essential bases for the future identification of the multiple mechanisms that underlie the therapeutic mode of action of MSC-CS and of the secretome components needed to repair $\mathrm{AD}$ brains. The tangible possibility, indeed, to subsequently manufacture these components will permit to prepare to-go biologic products opening a new horizon in the clinical use of MSC.

\section{Materials and methods}

\section{Mice}

APPswe/PS1 $\triangle \mathrm{e} 9$ (APP/PS1) transgenic male mice [B6C3$\mathrm{Tg}$ (APPswe,PSEN1dE9)85Dbo/Mmjax mice] and agematched wild type (WT) littermates were purchased from Jackson Laboratories (USA). No environmental enrichment was used since it notably improves AD pathology in mouse models of $\mathrm{AD}[55,56]$. Mice were all drug and behavioral test naïve and the experiments were all conducted during the light cycle. All animals were housed in a SPF facility in groups of 4 in standard mouse cages containing sawdust with food (2018S Envigo diet) and water ad libitum, under conventional laboratory conditions (room temperature: $20 \pm$ $2{ }^{\circ} \mathrm{C}$; humidity: $\left.60 \%\right)$ and a $12 / 12 \mathrm{~h}$ light/dark cycle $(7: 00$ am - 7:00 pm).

The IRFMN adheres to the principles set out in the following laws, regulation, and policies governing the Care and Use of Laboratory Animals: Italian Governing Law (D. lgs 26/2014; Authorization n.19/2008-A issued March 6, 2008 by Ministry of Health); Mario Negri Institutional Regulations and Policies providing internal authorization for persons conducting animal experiments (Quality 
Management System Certificate - UNI EN ISO 9001:2015 - Reg. $\mathrm{N}^{\circ}$ 6121); the NIH Guide for the Care and Use of Laboratory Animals (2011 edition) and EU directives and guidelines (EEC Council Directive 2010/63/UE). The statement of Compliance (Assurance) with the Public Health Service (PHS) Policy on Human Care and Use of Laboratory Animals has been reviewed (9/9/2014; Animal Welfare Assurance \#A5023-01).

\section{APP/PS1 brain homogenate preparation}

The brains of young or old WT, or those of APP/PS1 mice aged- and sex-matched to those undergoing the treatment were collected and homogenized on ice in DMEM $(100 \mathrm{mg} /$ $\mathrm{mL}$; GIBCO). The homogenate was thus centrifuged at $12.000 \mathrm{rcf}$ for $20 \mathrm{~min}$ at $4{ }^{\circ} \mathrm{C}$ and the supernatant collected and frozen in liquid nitrogen and stored at $-80^{\circ} \mathrm{C}$ until use.

\section{Preparation of MSC and secretome thereof}

Murine bone marrow-derived MSC were obtained and characterized as described previously [44], and cultured $\left(2 \times 10^{6}\right.$ cells in $10 \mathrm{~mL}$ medium in T75 flask) in supplemented Mesencult medium (StemCell Technologies) following manufacturer's instructions. MSC were negative for mycoplasma contamination. MSC were then incubated first in "starvation medium" (serum-free RPMI containing glutamine) for $30 \mathrm{~min}$, followed by stimulation with $10 \%$ APP/ PS1 mouse brain homogenate in Mesencult medium for 24 h. Upon removal of the stimulation medium, MSC were incubated in starvation medium for $24 \mathrm{~h}$, upon which the culture supernatant (MSC-CS) was collected and concentrated using Amicon Ultra centrifugal filters (Amicon Ultra-15, Millipore) to a volume equivalent to the secretome of $1 \times 10^{6} \mathrm{MSC}$ in $200 \mu \mathrm{l}$ for intravenous (IV) injection. Since the maximum intranasal (IN) administration volume is $25 \mu \mathrm{L}$, MSC-CS was concentrated by ultrafiltration to maintain the same IV MSC-CS dose (equivalent to $1 \times 10^{6}$ $\mathrm{MSC} / 200 \mu \mathrm{L}$ ) in $25 \mu \mathrm{L}$.

\section{MSC treatment and memory evaluation}

MSC were infused IV at $1 \times 10^{6}$ dose in a volume of 200 $\mu \mathrm{L}$. Mice were tested in the NORT 30 days post-injection.

\section{Experimental groups for MSC-CS treatment}

\section{Group 1}

Twelve-month-old APP/PS1 mice received one IV injection of either PBS $(n=6)$, MSC-CS $(n=5)$ or MSC-UCS $(n=$ $6)$. Seven days later were tested in the NORT. Age-matched WT received one IV injection of PBS $(n=7)$.

\section{Group 2}

Twelve-month-old APP/PS1 mice and age-matched WT received one IV injection of PBS or MSC-CS (WT + PBS $n=14 ; \quad \mathrm{WT}+\mathrm{MSC}-\mathrm{CS} n=6 ; \quad \mathrm{APP} / \mathrm{PS} 1+\mathrm{PBS} n=13$; APP/PS1 + MSC-CS $n=13$ ). Seven days later mice were tested in the NORT. Seven of them were then sacrificed for brain collection and neuropathological analysis the day after the test, the other were re-tested 14 days later to verify effect duration. This experiment was replicated twice.

\section{Group 3}

Twenty-two-month-old APP/PS1 mice received one IV injection of MSC-CS. Seven days post-injection they were tested in the NORT and the day after killed for brain collection and neuropathology assessment at a more advanced stage of disease compared to group 1 and 2 .

\section{Group 4}

Twenty-two month-old APP/PS1 and age-matched WT underwent a repeated intranasal (IN) treatment regimen (25 $\mu \mathrm{L} / \mathrm{inj}, 1$ inhalation/week) and were tested in the NORT 7 days after the first and fourth inhalation and again 30 days after the fourth inhalation (WT + PBS $n=6$; APP/PS1 + $\mathrm{PBS} n=7$; APP/PS1 + MSC-CS $n=9$ ). Mice were then retreated with 4 further weekly inhalations. Neuropathology was assessed 7 days after the eighth inhalation.

\section{Group 5 and 6}

Twenty-two-month-old and 25-month-old APP/PS1 and age-matched WT mice underwent a repeated IN treatment regimen $(25 \mu \mathrm{L} / \mathrm{inj}, 1$ inhalation/week) with MSC-CS collected from MSC exposed to brain homogenate derived from 20-month-old or 6-month-old WT mice, respectively. Mice were tested in the NORT 7 days after the fourth inhalation (22-month-old: WT + PBS $n=6$; APP/PS1 + PBS $n=6 ; \quad$ APP/PS1 + MSC-CS $n=6 ; 25$-month-old: $\mathrm{WT}+\mathrm{PBS} n=6 ; \quad \mathrm{APP} / \mathrm{PS} 1+\mathrm{PBS} n=7 ; \quad \mathrm{APP} / \mathrm{PS} 1+$ MSC-CS $n=6$ ).

\section{NORT}

Mice were tested in an open-square gray arena $(40 \times 40 \mathrm{~cm})$, $30 \mathrm{~cm}$ high as previously described [57]. The task started with a 5-min habituation trial. The next day, mice were again placed in the arena containing two identical objects (familiarization phase). Exploration was recorded in a 10min trial. Twenty-four hours later (test phase) mice were again placed in the arena containing two objects: one familiar and a new one, and the time spent exploring the 
Table 2 List of primers used for real-time reverse transcription polymerase chain reaction.

\begin{tabular}{lll}
\hline Gene & Forward Primer & Reverse Primer \\
\hline Bdnf & AGGCACTGGAACTCGCAATG & AAGGGCCCGAACATACGATT \\
Ngf & CCAAGGACGCAGCTTTCTAT & CCTGTACGCCGATCAAAAAC \\
Gap43 & CAGAGGATGCTGCCACCAA & GTTTGGCTTCGTCTACAGCG \\
Casp3 & GGTTCATCCAGTCCCTTTGC & CTAGCTTGTGCGCGTACAGC \\
\hline
\end{tabular}

two objects was recorded for $10 \mathrm{~min}$. Memory was expressed as a discrimination index (DI), i.e. (seconds on novel - seconds on familiar)/(total time on objects). Mice exposed to NORT for the second time were not subjected to the habituation trial.

\section{Immunohistochemistry for amyloid plaques, $\mathrm{A} \beta \mathrm{O}$ s or $\mathrm{CD} 68$}

Mice were anesthetized with a mixture of medetomidine/ ketamine and intracardially perfused with phosphate buffer saline (PBS); their brains were removed, post-fixed in $4 \%$ paraformaldehyde and stored at $-80^{\circ} \mathrm{C}$ after cryoprotection. APP/PS1 mouse brain coronal sections $(20 \mu \mathrm{m})$ were cut using a Leica cryostat and incubated for $1 \mathrm{~h}$ at room temperature (RT) with $10 \%$ normal goat serum (NGS) then overnight $(\mathrm{O} / \mathrm{N})$ at $4{ }^{\circ} \mathrm{C}$ with primary antibodies $(6 \mathrm{E} 10$ 1:500; Signet, Cat. \# 803001; A11 1:1000; Invitrogen, Cat. \# AHB0052; CD68 1:200; Biorad, Cat. \# MCA-1957). After incubation with the appropriate biotinylated secondary antibodies (1:200; Vector Laboratories), immunostaining was developed using the avidin-biotin kit (Vector Laboratories) and diaminobenzidine (Sigma, Italy) as chromogen. Only for the A11 staining DAB was amplified with nickel.

\section{Immunofluorescence}

Based on the analysis needed, $20 \mu \mathrm{m}$ APP/PS1 cryostat coronal brain sections were collected in $100 \mathrm{mM}$ PBS and co-immunostained with a maximum of 3 primary antibodies concomitantly. Plaques, CD68 and A11 were stained as described above. Microglial cells with anti-Iba1 (Iba1: 1:1000, O/N; Wako, Cat. \# 019-19741) and astrocytes with anti-GFAP (1:3500, O/N; Chemicon Int. Inc., Cat. \# MAB3402). We immunostained also IL-1 $\beta$ (1:200, Santa Cruz Biotechnology, Cat. \# SC-1252; $\left.72 \mathrm{~h}, 4{ }^{\circ} \mathrm{C}\right)$ and TNF $\alpha$ (1:100; Tebu-Bio, Cat. \# 500-P644-50UG; 72 h, $\left.4{ }^{\circ} \mathrm{C}\right)$ colocalized with astrocytes and microglia. These protocols were all previously described [32, 50]. The primary antibodies were followed by the application of the appropriate fluorescent secondary antibodies (1:500 for $1 \mathrm{~h}$ at RT) conjugated respectively with Alexa 488, 546 or 647 (Molecular Probes). In the case of IL-1 $\beta$ and TNF- $\alpha$ a streptavidin Tyramide Signal Amplification kit (NEN Life Science Products) was used with the secondary antibody.
Immunofluorescence was acquired using an IX81 microscope and a FV500 confocal scan unit with three laser lines [argon-krypton (488 nm), helium-neon red $(646 \mathrm{~nm})$, and helium-neon green (532 nm; Olympus)] and an ultraviolet diode.

\section{NISSL staining}

Twenty- $\mu \mathrm{m}$ slices were collected with a Leica cryostat on gelatin-coated slides for a total thickness of $760 \mu \mathrm{m}$ - from bregma -1.94 to -2.70 - selecting one slice every other 5 . After 2-day drying and a 1-min wash in $\mathrm{H}_{2} \mathrm{O}$, slices were dehydrated with $\mathrm{EtOH}(70 \%, 95 \%, 100 \%$, 5-min each), until xylene (5-min). Slices were processed backward until cresyl violet solution (3-min), followed by $\mathrm{H}_{2} \mathrm{O}+70 \%$ $\mathrm{EtOH}$ washes and 3-min in EtOH/acetic acid. Following $100 \% \mathrm{EtOH}$ and xylene (5-min/each), the slices were covered with a glass microcover (Prestige) using DPX mountant.

\section{Image analysis}

Quantitative analyses were done by an operator blind to genotype and treatment and normalizing on the quantified area. Area of selection for image analysis were the whole hippocampus and/or the whole cortex of a single hemisphere. We analyzed $320-\mu \mathrm{m}$ thick slices/mouse. Brain images were acquired using the Olympus Virtual Stage microscope. Immunofluorescence images were acquired using the Olympus confocal microscope; the acquisition setting was standardized for each analyzed marker and applied throughout the study. The brain area of interest was selected in bright field and the immunoreactivity for each specific marker was quantified by applying dedicated homemade macros through Fiji software [32, 50, 58].

\section{Gene expression analysis}

Total RNA was extracted from hippocampi by PureLink RNA Mini Kit (Invitrogen) following the manufacturer's instructions. Samples were treated with DNase I (Invitrogen) and reverse-transcribed with random hexamer primers using cDNA Reverse Transcription Kit (Applied Biosystem). The analysis was performed using Power syber green PCR master mix (Applied Biosystem) and 7300 Real-Time 
PCR System (Applied Biosystems). mRNA expression of target genes was evaluated using primers listed in Table 2. RPL27 was used as housekeeping gene to normalize mRNA levels. Relative gene expression was determined by $\Delta \Delta \mathrm{Ct}$ method. Data are expressed as $\log 2$ of the fold difference from the control group.

\section{Structured illumination microscopy (SIM)}

SIM analysis of brain sections was performed on a Nikon SIM system. Tissues were imaged at laser excitation of 488 (for A11), 561 (for CD68) nm with a 3D-SIM acquisition protocol. Sixteen-bit images sized $1024 \times 1024$ pixels with a single pixel of $0.030 \mu \mathrm{m}$ were acquired in a gray-level range of 0-16000 to exploit the linear range of the camera (iXon ultra DU-897U, Andor) and to avoid saturation. Threedimensional Z-stacks were scanned with a $0.125 \mu \mathrm{m}$ step size over 2-3 $\mu \mathrm{m}$. Raw and reconstructed images were validated with the SIM-check plugin of ImageJ [59] and only those providing satisfactory image diagnosis were included in the study. Images were finally elaborated with GIMP (Gnu Image Manipulation Program).

\section{Statistical analysis}

Data were expressed as scatter plots with mean \pm standard error of the mean (SEM) and analyzed using GraphPad Prism software. All data reported in the paper met the assumption of normal distribution. The estimated variance for NORT data was of 0.15 , whereas for histological biomarker analysis it was around 0.1. Notably, the variance we obtained was very similar between statistically compared experimental groups. We used either one-way or two-way analysis of variance (ANOVA) based on the experimental design. In the presence of a significant effect of treatment (one-way ANOVA), or a significant interaction between transgene and treatment (two-way ANOVA), appropriate post-hoc tests were applied. The Student $t$ test was used for a comparison between two experimental groups. A $P$ value $<0.05$ was considered significant. Sample size of 6-8 mice/ group was estimated on the basis of our wide experience with APP/PS1 mice. Experimental mouse groups with a lower number of mice (i.e. 4 or 5) are due to mouse exclusion or mouse death throughout the study. Exclusion criteria were pre-established as follows: (i) exclusion of mice from the NORT was applied only in the case of mouse immobility during either one of the test phases; (ii) the total time of object exploration was lower than $10 \mathrm{~s}$. Exclusion of samples for histological analysis was applied only in the case of brain tissue damage and inability to obtain quantifiable immuno-labeling. Mice were randomly selected from each cage to assign half of the animals to the control group and the other half to the treatment group. All the in-vivo and ex-vivo analyses were conducted in blind.

Acknowledgements We thank Dr Fumagalli for his contribution with imaging and Alessandro Soave for figure editing.

Funding This work was supported in part by the Paul G. Allen Family Foundation (USA).

\section{Compliance with ethical standards}

Conflict of interest The authors declare that they have no conflict of interest.

Publisher's note Springer Nature remains neutral with regard to jurisdictional claims in published maps and institutional affiliations.

Open Access This article is licensed under a Creative Commons Attribution 4.0 International License, which permits use, sharing, adaptation, distribution and reproduction in any medium or format, as long as you give appropriate credit to the original author(s) and the source, provide a link to the Creative Commons license, and indicate if changes were made. The images or other third party material in this article are included in the article's Creative Commons license, unless indicated otherwise in a credit line to the material. If material is not included in the article's Creative Commons license and your intended use is not permitted by statutory regulation or exceeds the permitted use, you will need to obtain permission directly from the copyright holder. To view a copy of this license, visit http://creativecommons. org/licenses/by/4.0/.

\section{References}

1. Selkoe DJ, Hardy J. The amyloid hypothesis of Alzheimer's disease at 25 years. EMBO Mol Med. 2016;8:595-608.

2. Selkoe DJ. Alzheimer's disease. Cold Spring Harb Perspect Biol. 2011,3:a004457.

3. Selkoe DJ. Alzheimer's disease is a synaptic failure. Science. 2002;298:789-91.

4. Benilova I, Karran E, De Strooper B. The toxic Abeta oligomer and Alzheimer's disease: an emperor in need of clothes. Nat Neurosci. 2012;15:349-57.

5. Heneka MT, Carson MJ, El Khoury J, Landreth GE, Brosseron F, Feinstein DL, et al. Neuroinflammation in Alzheimer's disease. Lancet Neurol. 2015;14:388-405.

6. Balducci C, Forloni G. Novel targets in Alzheimer's disease: a special focus on microglia. Pharmacol Res. 2018;130:402-13.

7. Uccelli A, Benvenuto F, Laroni A, Giunti D. Neuroprotective features of mesenchymal stem cells. Best Pr Res Clin Haematol. 2011;24:59-64.

8. Fan X, Sun D, Tang X, Cai Y, Yin ZQ, Xu H. Stem-cell challenges in the treatment of Alzheimer's Disease: a long way from bench to bedside. Med Res Rev. 2014;34:957-78.

9. Duncan T, Valenzuela M. Alzheimer's disease, dementia, and stem cell therapy. Stem Cell Res Ther. 2017;8:111.

10. Laroni A, Novi G, Kerlero de Rosbo N, Uccelli A. Towards clinical application of mesenchymal stem cells for treatment of neurological diseases of the central nervous system. J Neuroimmune Pharm. 2013;8:1062-76.

11. Abbasi-Malati Z, Roushandeh AM, Kuwahara Y, Roudkenar MH. Mesenchymal stem cells on horizon: a new arsenal of therapeutic agents. Stem Cell Rev. 2018;14:484-99. 
12. Teixeira FG, Carvalho MM, Sousa N, Salgado AJ. Mesenchymal stem cells secretome: a new paradigm for central nervous system regeneration? Cell Mol Life Sci. 2013;70:3871-82.

13. Balducci C, Frasca A, Zotti M, La Vitola P, Mhillaj E, Grigoli E, et al. Toll-like receptor 4-dependent glial cell activation mediates the impairment in memory establishment induced by $\beta$-amyloid oligomers in an acute mouse model of Alzheimer's disease. Brain Behav Immun. 2017;60:188-97.

14. Itagaki S, McGeer PL, Akiyama H, Zhu S, Selkoe D. Relationship of microglia and astrocytes to amyloid deposits of Alzheimer disease. J Neuroimmunol. 1989;24:173-82.

15. Cohen SJ, Stackman RW Jr. Assessing rodent hippocampal involvement in the novel object recognition task. A review. Behav Brain Res. 2015;285:105-17.

16. Perego C, Fumagalli S, Zanier ER, Carlino E, Panini N, Erba E, et al. Macrophages are essential for maintaining a M2 protective response early after ischemic brain injury. Neurobiol Dis. 2016;96:284-93.

17. Holness CL, Simmons DL. Molecular cloning of CD68, a human macrophage marker related to lysosomal glycoproteins. Blood. 1993;81:1607-13.

18. Trotta T, Porro C, Calvello R, Panaro MA. Biological role of Tolllike receptor-4 in the brain. J Neuroimmunol. 2014;268:1-12.

19. Liddelow SA, Guttenplan KA, Clarke LE, Bennett FC, Bohlen CJ, Schirmer L, et al. Neurotoxic reactive astrocytes are induced by activated microglia. Nature. 2017;541:481-7.

20. Galeano C, Qiu Z, Mishra A, Farnsworth SL, Hemmi JJ, Moreira A, et al. The route by which intranasally delivered stem cells enter the central nervous system. Cell Transpl. 2018;27:501-14.

21. Barrientos RM, Kitt MM, Watkins LR, Maier SF. Neuroinflammation in the normal aging hippocampus. Neuroscience. 2015;309:84-99.

22. Valles SL, Iradi A, Aldasoro M, Vila JM, Aldasoro C, de la Torre $\mathrm{J}$, et al. Function of glia in aging and the brain diseases. Int $\mathrm{J}$ Med Sci. 2019;16:1473-9.

23. Forloni G, Artuso V, La Vitola P, Balducci C. Oligomeropathies and pathogenesis of Alzheimer and Parkinson's diseases. Mov Disord J Mov Disord Soc. 2016;31:771-81.

24. Forloni G, Balducci C. Alzheimer's disease, oligomers, and inflammation. J Alzheimers Dis JAD. 2018;62:1261-76.

25. Micotti E, Paladini A, Balducci C, Tolomeo D, Frasca A, Marizzoni $\mathrm{M}$, et al. Striatum and entorhinal cortex atrophy in AD mouse models: MRI comprehensive analysis. Neurobiol Aging. 2015;36:776-88.

26. Venneri A, Gorgoglione G, Toraci C, Nocetti L, Panzetti P, Nichelli P. Combining neuropsychological and structural neuroimaging indicators of conversion to Alzheimer's disease in amnestic mild cognitive impairment. Curr Alzheimer Res. 2011;8:789-97.

27. Grayson B, Leger M, Piercy C, Adamson L, Harte M, Neill JC. Assessment of disease-related cognitive impairments using the novel object recognition (NOR) task in rodents. Behav Brain Res. 2015;285:176-93.

28. Cui G-H, Wu J, Mou F-F, Xie W-H, Wang F-B, Wang Q-L, et al. Exosomes derived from hypoxia-preconditioned mesenchymal stromal cells ameliorate cognitive decline by rescuing synaptic dysfunction and regulating inflammatory responses in APP/PS1 mice. FASEB. J Publ Fed Am Soc Exp Biol. 2018;32:654-68.

29. Duyckaerts C, Delatour B, Potier MC. Classification and basic pathology of Alzheimer disease. Acta Neuropathol. 2009;118:5-36.

30. Morris GP, Clark IA, Zinn R, Vissel B. Microglia: a new frontier for synaptic plasticity, learning and memory, and neurodegenerative disease research. Neurobiol Learn Mem. 2013;105:40-53.
31. Ota Y, Zanetti AT, Hallock RM. The role of astrocytes in the regulation of synaptic plasticity and memory formation. Neural Plast. 2013;2013:185463.

32. Zanier ER, Pischiutta F, Riganti L, Marchesi F, Turola E, Fumagalli $\mathrm{S}$, et al. Bone marrow mesenchymal stromal cells drive protective M2 microglia polarization after brain trauma. Neurotherapeutics. 2014;11:679-95.

33. Shin JY, Park HJ, Kim HN, Oh SH, Bae J-S, Ha H-J, et al. Mesenchymal stem cells enhance autophagy and increase $\beta$ amyloid clearance in Alzheimer disease models. Autophagy. 2014;10:32-44.

34. Zuroff L, Daley D, Black KL, Koronyo-Hamaoui M. Clearance of cerebral $\mathrm{A} \beta$ in Alzheimer's disease: reassessing the role of microglia and monocytes. Cell Mol Life Sci CMLS. 2017;74:2167-201.

35. Hong S, Beja-Glasser VF, Nfonoyim BM, Frouin A, Li S, Ramakrishnan S, et al. Complement and microglia mediate early synapse loss in Alzheimer mouse models. Science. 2016;352:712-6.

36. Li S, Selkoe DJ. A mechanistic hypothesis for the impairment of synaptic plasticity by soluble $\mathrm{A} \beta$ oligomers from Alzheimer's brain. J. Neurochem. 2020; https://doi.org/10.1111/ jnc. 15007.

37. Li Y, Feng L, Zhang G-X, Ma C. Intranasal delivery of stem cells as therapy for central nervous system disease. Exp Mol Pathol. 2015;98:145-51.

38. Salama M, Sobh M, Emam M, Abdalla A, Sabry D, El-Gamal M, et al. Effect of intranasal stem cell administration on the nigrostriatal system in a mouse model of Parkinson's disease. Exp Ther Med. 2017;13:976-82.

39. Long Q, Upadhya D, Hattiangady B, Kim D-K, An SY, Shuai B, et al. Intranasal MSC-derived A1-exosomes ease inflammation, and prevent abnormal neurogenesis and memory dysfunction after status epilepticus. Proc Natl Acad Sci U S A. 2017;114: E3536-45.

40. Oppliger B, Joerger-Messerli M, Mueller M, Reinhart U, Schneider P, Surbek DV, et al. Intranasal delivery of umbilical cord-derived mesenchymal stem cells preserves myelination in perinatal brain damage. Stem Cells Dev. 2016;25:1234-42.

41. Uccelli A, de Rosbo NK. The immunomodulatory function of mesenchymal stem cells: mode of action and pathways. Ann N Y Acad Sci. 2015;1351:114-26.

42. Krampera M, Cosmi L, Angeli R, Pasini A, Liotta F, Andreini A, et al. Role for interferon-gamma in the immunomodulatory activity of human bone marrow mesenchymal stem cells. Stem Cells Dayt Ohio. 2006;24:386-98.

43. Saeedi P, Halabian R, Fooladi AAI. Mesenchymal stem cells preconditioned by staphylococcal enterotoxin $\mathrm{B}$ enhance survival and bacterial clearance in murine sepsis model. Cytotherapy. 2019;21:41-53.

44. Zappia E, Casazza S, Pedemonte E, Benvenuto F, Bonanni I, Gerdoni E, et al. Mesenchymal stem cells ameliorate experimental autoimmune encephalomyelitis inducing T-cell anergy. Blood. 2005;106:1755-61.

45. Cedola A, Bravin A, Bukreeva I, Fratini M, Pacureanu A, Mittone A, et al. X-ray phase contrast tomography reveals early vascular alterations and neuronal loss in a multiple sclerosis model. Sci Rep. 2017;7:5890.

46. Bai L, Lennon DP, Caplan AI, DeChant A, Hecker J, Kranso J, et al. Hepatocyte growth factor mediates mesenchymal stem cellinduced recovery in multiple sclerosis models. Nat Neurosci. 2012;15:862-70

47. Uccelli A, Milanese M, Principato MC, Morando S, Bonifacino T, Vergani L, et al. Intravenous mesenchymal stem cells improve 
survival and motor function in experimental amyotrophic lateral sclerosis. Mol Med. 2012;18:794-804.

48. Mucke L, Selkoe DJ. Neurotoxicity of amyloid beta-protein: synaptic and network dysfunction. Cold Spring Harb Perspect Med. 2012;2:a006338.

49. Balducci C, Forloni G. In vivo application of beta amyloid oligomers: a simple tool to evaluate mechanisms of action and new therapeutic approaches. Curr Pharm Des. 2014;20:2491-505.

50. Balducci C, Santamaria G, La Vitola P, Brandi E, Grandi F, Viscomi AR, et al. Doxycycline counteracts neuroinflammation restoring memory in Alzheimer's disease mouse models. Neurobiol Aging. 2018;70:128-39.

51. Hampel H, Bürger K, Teipel SJ, Bokde ALW, Zetterberg H, Blennow K. Core candidate neurochemical and imaging biomarkers of Alzheimer's disease. Alzheimers Dement J Alzheimers Assoc. 2008;4:38-48.

52. Delbeuck X, Van der Linden M, Collette F. Alzheimer's disease as a disconnection syndrome? Neuropsychol Rev. 2003;13:79-92.

53. Zilka N, Zilkova M, Kazmerova Z, Sarissky M, Cigankova V, Novak M. Mesenchymal stem cells rescue the Alzheimer's disease cell model from cell death induced by misfolded truncated tau. Neuroscience. 2011;193:330-7.
54. Oh SH, Kim HN, Park H-J, Shin JY, Lee PH. Mesenchymal stem cells increase hippocampal neurogenesis and neuronal differentiation by enhancing the Wnt signaling pathway in an Alzheimer's disease model. Cell Transpl. 2015;24:1097-109.

55. Lazarov O, Robinson J, Tang YP, Hairston IS, Korade-Mirnics Z, Lee VM, et al. Environmental enrichment reduces Abeta levels and amyloid deposition in transgenic mice. Cell. 2005;120:701-13.

56. Valero J, Espana J, Parra-Damas A, Martin E, Rodriguez-Alvarez J, Saura CA. Short-term environmental enrichment rescues adult neurogenesis and memory deficits in $\mathrm{APP}(\mathrm{Sw}, \mathrm{Ind})$ transgenic mice. PLoS One. 2011;6:e16832.

57. Balducci C, Beeg M, Stravalaci M, Bastone A, Sclip A, Biasini E, et al. Synthetic amyloid-beta oligomers impair long-term memory independently of cellular prion protein. Proc Natl Acad Sci USA. 2010;107:2295-300.

58. Schindelin J, Arganda-Carreras I, Frise E, Kaynig V, Longair M, et al. Fiji: an open-source platform for biological-image analysis. Nat Methods. 2012;9:676-82.

59. Ball G, Demmerle J, Kaufmann R, Davis I, Dobbie IM, Schermelleh L. SIMcheck: a toolbox for successful super-resolution structured illumination microscopy. Sci Rep. 2015;5:15915. 